\title{
The Role of Fructose in Public Health and Obesity
}

\author{
Carla Torres Carvalho', Zelia Maria Souza1, Nawal Arbex², Diana Sá1, \\ Luciana Corrêa de Souza Rodrigues', Diana Aristotelis Rocha de Sá1, \\ Larissa Bianca Paiva Cunha de Sá1, Alberto Krayyem Arbex ${ }^{1,3,4}$ \\ ${ }^{1}$ Endocrinology Division, IPEMED Medical School, Rio de Janeiro, Brazil \\ ${ }^{2}$ Gastronomy Faculty, Centro de Ensino Superior, Juiz de Fora, Brazil \\ ${ }^{3}$ Diabetology Department, Malteser Krankenhaus St. Franziskus Hospital, Flensburg, Germany \\ ${ }^{4}$ Harvard T. H. Chan School of Public Health, Boston, MA, USA \\ Email: ctc.carlatorres@gmail.com
}

How to cite this paper: Carvalho, C.T., Souza, Z.M., Arbex, N., Sá, D., de Souza Rodrigues, L.C., de Sá, D.A.R., de Sá, L.B.P.C. and Arbex, A.K. (2018) The Role of Fructose in Public Health and Obesity. Health, 10, 434-441.

https://doi.org/10.4236/health.2018.104035

Received: February 27, 2018

Accepted: April 24, 2018

Published: April 27, 2018

Copyright $\odot 2018$ by authors and Scientific Research Publishing Inc. This work is licensed under the Creative Commons Attribution International License (CC BY 4.0).

http://creativecommons.org/licenses/by/4.0/

\begin{abstract}
We are in the so-called nutritional transition, in which obesity and its comorbidities have emerged as an important research topic, and the information on food composition is fundamental to promote nutritional safety. Fructose is the sweetest carbohydrate, and this sweetness, along with its low cost, is the key factor for its use in commercial drinks and sweets. The global average consumption of fructose per capita has increased from $56 \mathrm{~g} /$ day in 1986 to 65 g/day in 2007. Experimental models associate high fructose intake with the development of obesity and induced insulin resistance. In recent studies, diabetic patients have been reported to use fructose more frequently than glucose. Fructose acts differently in the hypothalamus and generates less satiety than glucose; thus, fructose has a high lipogenic potential. Replacing fructose with another isocaloric carbohydrate is associated with better glycemic control.
\end{abstract}

\section{Keywords}

Fructose, Obesity, Type 2 Diabetes, Food Composition, Carbohydrate

\section{Introduction}

Fructose is a monosaccharide that naturally present in food, such as fruits, sugar cane, honey, beetroot, and other foods. For its sweet taste and low cost, in the 1970s, glucose syrup, better known as high fructose corn syrup (HFCS), was introduced for manufactured products that use liquid sugar. In the first generation of HFCSs, there was $42 \%$ fructose in the formula. In the second generation, de- 
veloped in 1976, the percentage of fructose varied between $55 \%$ and $90 \%$ of the compounds [1].

In the USA, the soft drink industry accounted for $71 \%$ of the total HFCS consumption in 1987, since fructose is abundantly present in liquid form. At the time, the use of fructose in the soft drink market reached $96 \%$ of the total use, namely $66 \%$ in canned and processed foods, $35 \%$ in dairy products, $25 \%$ in baking products, and $3 \%$ in confectionery and non-food uses. Fructose consumption increased to approximately one million tons in 1985, since the product was used by major soft drink companies, represented $100 \%$ of their sweeteners, and led to an increase of almost $23 \%$ in one year [1].

\section{Fructose and Comorbidities}

In the USA, the annual consumption per capita of non-diet drinks increased 86\% between 1970 and 1997 [2] [3], and the prevalence of obesity increased by $112 \%$ during the same time. This leads us to consider whether the two factors are linked [2] [4]. Energy consumption per capita of added sugar increased from $984 \mathrm{~kJ}$ ( $235 \mathrm{kcal}$ ) per day between 1977 and 1978 to $1331 \mathrm{~kJ}$ (318 kcal) between 1994 and 1996, with soft drinks contributing much more to the total than other products, such as fruit drinks and desserts, despite being just as harmful [2] [5]. This large-scale increase in the production and consumption of fructose is not only observed in the USA, but also worldwide. Increased production and consumption of fructose has been observed at smaller scales in some countries, due to fees imposed by each country to regulate its commercialization [1].

One of the most consistent findings is the association between the intake of soft drinks and the increase in overall energy consumption. Soft drinks (sweetened with HFCS) are amongst the primary contributors to obesity and its comorbidities, particularly among children. In one of the studies analyzed, children and adolescents consumed between 122 and $159 \mathrm{~g}$ of added sugar, although the Department of Agriculture in the USA recommends using only 32 grams. Children that consume a soft drink per day consume $10 \%$ more calories per day than children that do not consume soft drinks. Over $70 \%$ of adolescents consume soft drinks daily in the UK [2] [6] [7]. All five longitudinal studies that were analyzed in the meta-analysis entitled Effects of Soft Drink Consumption on Nutrition and Health identified positive associations between soft drink consumption and overall energy intake [2]. Of the studies analyzed, 10 of 12 cross-sectional studies, all five longitudinal studies, and the four long-term experimental studies showed that energy intake is directly related to the consumption of fructose-sweetened drinks. The excess energy from the ingested fructose is not properly compensated in other foods, leading to an increase in the total energy. Studies suggest that the consumption of soft drinks sweetened with HFCS increases hunger, decreases satiety, and changes taste to high sweetness levels, changing food preference [2] [6]. According to the World Health Organization (WHO), 39\% of the adults worldwide were overweight in 2014, and the world- 
wide prevalence of obesity increased to over half a billion adults. In turn, 42 million children under five years of age were overweight in 2015, almost half of whom were in Africa and Asia.

The increase in carbohydrate intake, associated with the consumption of soft drinks with HFCS, causes a higher consumption of added sugars, when macronutrients and micronutrients are considered. An association with Fator vitamin A and B 12 intake could not be established [2] [6], but excess fructose consumption was associated with decreased protein intake, riboflavin, and development of hypocalcemia [2] [8] [9]. One of the studies performed a 30-day follow-up in patients with hypocalcemia and no consumption of soft drinks during this period, over which a significant increase in serum calcium was observed [2] [9]. Another two studies reported a statistically significant association between soft drink consumption and bone mineral density [2] [10] [11].

The strongest association between fructose-sweetened soft drink consumption and health outcomes is supported by the prospective evidence obtained for type 2 diabetes. According to the WHO, the number of people with diabetes increased from 108 million in 1980 to 422 million in 2014, and type 2 diabetes accounted for 1.5 million deaths in 2012. A total of 9129 women were followed for eight years in a study that was published in JAMA in 2004, which showed that women consuming one or more portions of fructose-sweetened soft drinks per day had two times higher risk of developing type 2 diabetes throughout the study than those consuming less than one portion per month [2] [12]. Another study reported a positive association between soft drink consumption and the number of metabolic syndrome risk factors. These effects were also recorded when the body mass indices (BMIs) and the amount of energy ingested were controlled [2] [13].

Bray et al. observed that Americans over the age of two years consume $553 \mathrm{~kJ}$ (132 kcal) per day from corn syrup (fructose), which is the only sweetener in the USA soft drinks, and that consumption of this sweetener increased by $1000 \%$ between 1970 and 1990. Fructose is proposedly digested, absorbed, and metabolized differently than glucose, promoting lipogenesis and not stimulating insulin secretion or increased leptin production, which are both afferent signals in the regulation of food intake and body weight [2] [14] [15].

\section{Differences between Fructose and Glucose}

Fructose consumption for 10 weeks at a dose equivalent to $25 \%$ of the required energy increased lipogenesis and visceral adiposity and promoted dyslipidemia and insulin resistance in overweight or obese women and men. This was not observed with glucose consumption, although the final weight gain was identical. It can thus be concluded that fructose and glucose have different metabolic effects that are independent of weight gain [6] [15].

Lipid metabolism and insulin sensitivity are known to be affected by body weight and body fat distribution, but little is known about the modulation that 
occurs in response to dietary macronutrients [15]. Some studies have been performed to elucidate this response, especially regarding ingested sugars. Two hypotheses on the effects of fructose consumption were investigated in a study that was published in December 2017: the endocrine effects add to a positive energy balance and the development of an atherogenic lipid profile. Previous short- and long-term studies have shown important differences between glucose and fructose consumption in humans. Excess fructose, both in normal weight women and in overweight or obese men and women, led to lower plasma concentrations of glucose, insulin, and leptin, and significantly increased triacylglycerol concentrations. In addition, postprandial ghrelin suppression was observed, and long-term studies showed higher concentrations of apolipoprotein B. Fructose-sweetened soft drink consumption also reduced the differences between leptin concentrations in the morning (lowest) and the in the evening (highest) in a short-term study of overweight and obese individuals [15] [16]. The suggested explanation was that leptin production is regulated by insulin-mediated glucose metabolism. Increased fructose intake does not increase glucose and insulin concentrations, and leptin concentration is reduced.

Insulin and leptin are responsible for endocrine signaling in the central nervous system (CNS) in the long-term regulation of energy balance. Prolonged high fructose consumption could lead to a daily caloric increase and reduced energy expenditure, contributing to weight gain and obesity that are caused by reduced insulin and leptin signaling in the CNS [15] [16]. However, while it is difficult to obtain such evidence in humans, an 8-week follow-up study of men and women, with BMIs between 25 and 35, showed that leptin concentrations were reduced in 24 hours, compared to glucose intake, but no differences in energy intake or weight gain were observed between the groups [15]. The long-term study in Rhesus monkeys, as an attempt to better elucidate this issue, also presented controversial results; and thus, more long-term interventional studies in humans are required [16].

\section{Fructose Generates an Atherogenic Profile}

Regarding the lipid profile, the results of long-term studies have shown that fructose-sweetened soft drink consumption increases triacylglycerol levels. De novo hepatic lipogenesis increases with the ingestion of fructose, since the liver where fructose is mainly metabolized. In hepatocytes, fructose is absorbed through GLUT-5 and enters glycolysis via fructose 1-phosphate, disregarding the main step that controls the rate of glucose metabolism, which is catalyzed by phosphofructokinase. The glycolysis of fructose provides unregulated amounts of acetyl-CoA and glycerol3-phosphate. Fructose 1-phosphate may be converted to glyceraldehyde or dihydroxyacetonephosphate, while glyceraldehyde is converted to glyceraldehyde 3-phosphate, which is then converted to pyruvate. Pyruvate enters the cellular respiration chain or produces acetyl-CoA, which will provide carbon skeletons for the synthesis of acyl-coenzyme A (acyl-CoA). The 
dihydroxyacetone phosphate will be converted into glycerol 3-phosphate, which will bind to acyl-CoA molecules and then convert to acyl glycerol, which is used for the synthesis of triglycerides or, through the addition of an ApoB molecule, to produce very low density lipoprotein (VLDL). Fructose upregulates the insulin-dependent SREBP-1c receptor, activating genes involved in de novo lipogenesis, fatty acid synthesis, and acetyl-CoA carboxylase [15] [16] [17] (Figure 1).

Growing evidence has shown an association between postprandial lipemia and the pro-atherogenic condition, and Apo B is clinically important. Long-term studies have shown increased numbers and reduced volumes of low density lipoprotein (LDL) particles. Consequently, the conformation of Apo B changes, increasing its affinity for proteoglycans of the arterial wall. It can thus be concluded that prolonged fructose consumption produces an atherogenic profile [16].

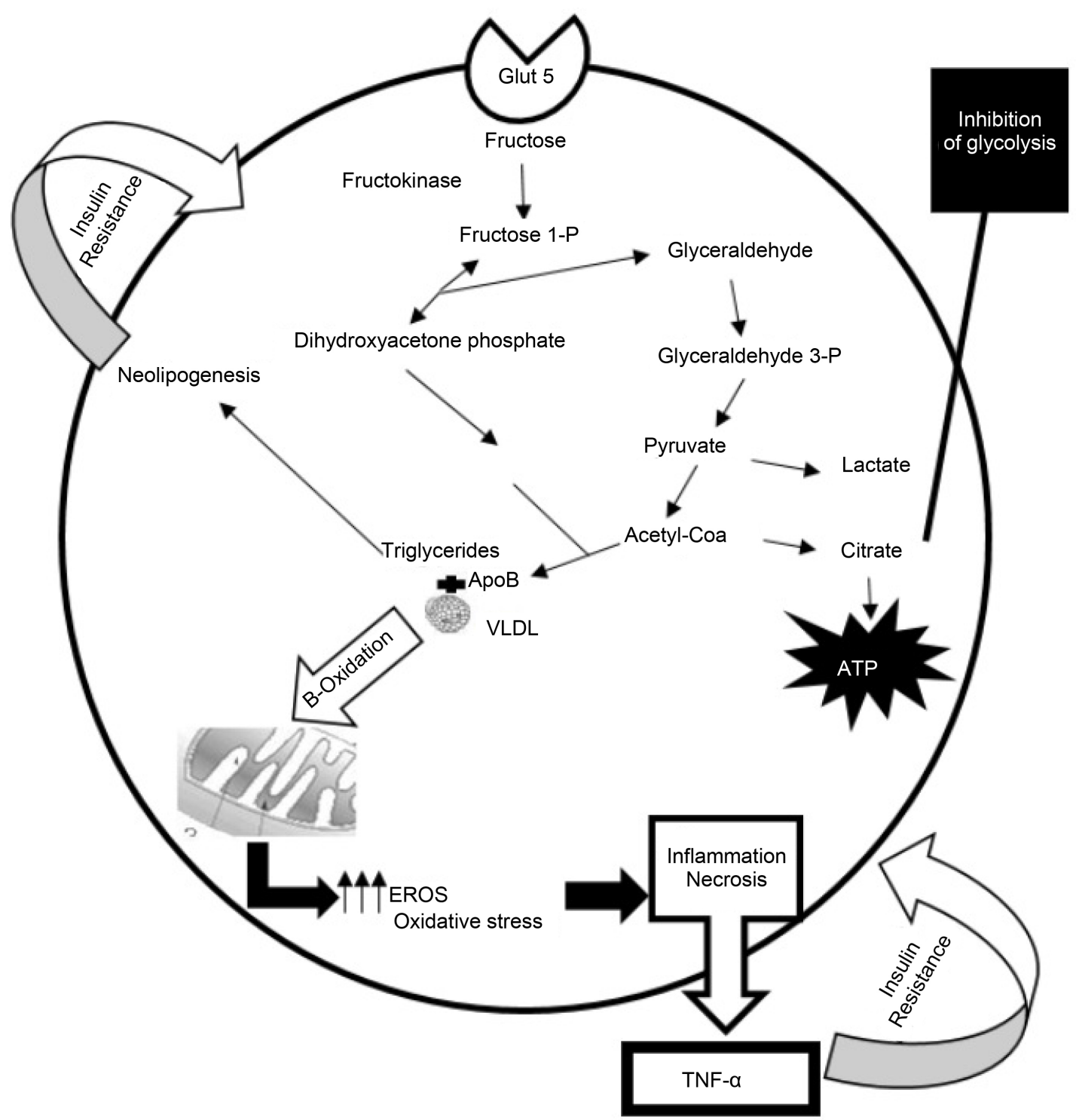

Figure 1. Mechanism of insulin resistance caused by excess fructose consumption [17]. 


\section{Strong Association with Hepatic Steatosis and Metabolic Syndrome}

Nonalcoholic steatohepatitis (NAHS) refers to hepatic steatosis and steatohepatitis, and to possible outcomes such as fibrosis, cirrhosis, and liver failure. NAHS is present in $2.6 \%$ of children and $26 \%$ of adults. The onset of this disease has a $50 \%$ correlation with the presence of type 2 diabetes and a $76 \%$ correlation with obesity. When associated with morbid obesity and type 2 diabetes, it is present in $95 \%$ of the cases [17].

Studies have shown a correlation between diets with a high fructose consumption and elevations of the aspartate aminotransferase (AST), alanine aminotransferase (ALT), and alkaline phosphatase (ALK), which are reliable predictors metabolic syndrome development. For better elucidation, a high fructose diet was introduced in rats and the outcomes included moderate hypertension, glucose intolerance, insulin resistance, hyperinsulinemia, and hypertriglyceridemia [17].

Energy production through fructose increases citrate concentrations in the mitochondria, and citrate is a potent phosphofructokinase (PFK) inhibitor. PFK is the allosteric modulator of glucose, inhibiting glycolysis and decreasing glucose participation in energy production. Hepatocytes increase energy production based on stored TG, which would contribute to hepatic resistance to insulin action. In addition, increased lipid oxidation enhances beta oxidation, which is a potent producer of reactive oxygen species that enhances hepatic oxidative stress. An inflammatory process occurs and causes hepatocyte apoptosis and hepatic necrosis in the long term. Important inflammation signals are released into the bloodstream. One of them, TNF- $\alpha$, is a potent inhibitor of insulin action, and a potent inhibitor of the production and action of adiponectin, an important hormone for insulin sensitivity. Thus, in the long term, high fructose intake generates metabolic syndromes [17].

\section{Health Measures}

In Geneva, January 28 to February 1, 2002, experts gathered in the so-called Joint WHO/FAO Expert Consultation on Diet, Nutrition and the Prevention of Chronic Diseases. The recommendation for free sugar consumption was $<10 \%$ of the total energy. Since the provision of this information, in November 2003, the "World Declaration and Plan of Action for Nutrition" was implemented, and many countries have adhered to this plan of action to improve national food and quality of life. Each country would consider macro and micronutrient consumption goals, as well as energy consumption goals, when formulating national nutritional policies. It is not an easy measure, as it requires total political commitment and interactions with all interested parties [6].

School programs that have been designed to stimulate physical exercise and diet changes are mostly ineffective, due to poor adherence to diets without free sugars in the child and adolescent populations. In the United Kingdom, the 
APPLES (Active Programme Prompting Lifestyle in Schools) program, which includes teacher training, changes in school meals, curriculum action plans, physical education and playground activities, generated a slight increase in the consumption of vegetables and non-sugar products, without changing the percentage of obesity in children [7].

The Director of the Department of Prevention of Noncommunicable Diseases of the WHO, Douglas Bettcher, says that the consumption of free sugars is an important factor for the global increase in the number of people suffering from obesity and diabetes, and that strategies should be implemented to reduce consumption. A possible action is the inclusion of fees in high fructose products, to stimulate a potential substitution by consumers. Some groups, especially those with low incomes and young people, are more responsive to changes in product prices. As such, some countries adopted fiscal measures for protection. For example, Mexico and Hungary set taxes on products with high sugar levels. Soft drinks have been banned or severely reduced in schools in Britain, France, Los Angeles, Philadelphia, and Miami [2].

\section{Conclusions}

Excessive fructose consumption increases every year, as well as its comorbidities. The most obvious fructose effect is the increase in overall energy consumption, which heavily contributes to the onset of obesity, especially in children. Statistically, fructose appears prevalent in the development of type 2 diabetes. The other striking effect for public health is the increased cardiovascular risk related to changes in lipogenesis.

More studies are required to further elucidate the effects of fructose consumption, but the existing studies show its profound impact on society and public health. Thus, the introduction of preventive measures is necessary.

\section{References}

[1] Yoshii, R.J., Carvalho, F.C.C. and Veiga Filho, A.A. (1994) Current Situation and Prospects of the World Market of Corn Syrup with High Fructose Content (HFCS). [Situacao atual e perspectivas do mercado mundial de xarope de milho de alto teor de frutose (HFCS).] Informações Econômicas SP, 24, 1-8.

[2] Vartanian, L.R., Schwartz, M.B. and Brownell, K.D. (2007) Effects of Soft Drink Consumption on Nutrition and Health: A Systematic Review and Meta-Analysis. American Public Health Association, 97, 4. https://doi.org/10.2105/AJPH.2005.083782

[3] Putnam, J.J. and Allshouse, J.E. (1999) Food Consumption, Prices and Expenditures, 1970-1997. Economic Research Service, US Department of Agriculture, Washington DC.

[4] Flegal, K.M., Carroll, M.D., Ogden, C.L. and Johnson, C.L. (2002) Prevalence and Trends in Obesity among US Adults, 1999-2000. JAMA, 288, 1723-1727. https://doi.org/10.1001/jama.288.14.1723

[5] Popkin, B.M. and Nielsen, S.J. (2003) The Sweetening of the World's Diet. Obesity Research, 11, 1325-1332. https://doi.org/10.1038/oby.2003.179 
[6] Nishida, C., Uauy, R., Kumanyika, S. and Shetty, P. (2004) The Joint WHO/FAO Expert Consultation on Diet, Nutrition and the Prevention of Chronic Diseases: Process, Product and Policy Implications. Public Health Nutrition, 7, 245-250. https://doi.org/10.1079/PHN2003592

[7] James, J., et al. (2004) Preventing Childhood Obesity Reducing Consumption of Carbonated Drinks: Cluster Randomized Controlled Trial. BMJ, 328, 1237. https://doi.org/10.1136/bmj.38077.458438.EE

[8] Guerrero-Romero, F., Rodriguez-Moran, M. and Reyes, E. (1999) Consumption of Soft Drinks with Phosphoric Acid as a Risk Factor for the Development of Hypocalcemia in Postmenopausal Women. Journal of Clinical Epidemiology, 52, 1007-1010. https://doi.org/10.1016/S0895-4356(99)00097-9

[9] Mazariegos-Ramos, E., Guerrero-Romero, F., Rodriguez-Moran, M., Lazcano-Burciaga, G., Paniagua, R. and Amato, D. (1995) Consumption of Soft Drinks with Phosphoric Acid as a Risk Factor for the Development of Hypocalcemia in Children: A Case-Control Study. Journal of Pediatrics, 126, 940-942. https://doi.org/10.1016/S0022-3476(95)70215-6

[10] Whiting, S.J., Healey, A., Psiuk, S., Mirwald, R., Kowalski, K. and Bailey, D.A. (2001) Relationship between Carbonated and Other Low Nutrient Dense Beverages and Bone Mineral Content of Adolescents. Nutrition Research, 21, 1107-1115. https://doi.org/10.1016/S0271-5317(01)00324-4

[11] McGartland, C., Robson, P.J., Murray, L., et al. (2003) Carbonated Soft Drink Consumption and Bone Mineral Density in Adolescence: The Northern Ireland Young Hearts Project. Journal of Bone and Mineral Research, 18, 1563-1569. https://doi.org/10.1359/jbmr.2003.18.9.1563

[12] Schulze, M.B., Manson, J.E., Ludwig, D.S., et al. (2004) Sugarsweetened Beverages, Weight Gain, and Incidence of Type 2 Diabetes in Young and Middle-Aged Women. JAMA, 292, 927-934. https://doi.org/10.1001/jama.292.8.927

[13] Yoo, S., Nicklas, T., Baranowski, T., et al. (2004) Comparison of Dietary Intakes Associated with Metabolic Syndrome Risk Factors in Young Adults: The Bogalusa Heart Study. American Journal of Clinical Nutrition, 80, 841-848. https://doi.org/10.1093/ajcn/80.4.841

[14] Bray, G.A., Nielsen, S.J. and Popkin, B.M. (2004) Consumption of High-Fructose Corn Syrup in Beverages May Play a Role in the Epidemic of Obesity. American Journal of Clinical Nutrition, 79, 537-543. https://doi.org/10.1093/ajcn/79.4.537

[15] Cianflone, K., McGahan, J.P., Berglund, L., Bremer, A.A., Keim, N.L., Griffen, S.C., Havel, P.J. and Stanhope, K.L. (2013) Effects of Sugar-Sweetened Beverages on Plasma Acylation Stimulating Protein, Leptin and Adiponectin: Relationships with Metabolic Outcomes. Journal of Obesity, 21, 12.

[16] Stanhope, K.L. and Havel, P.J. (2008) Endocrine and Metabolic Effects of Consuming Beverages Sweetened with Fructose, Glucose, Sucrose, or High-Fructose Corn Syrup. American Journal of Clinical Nutrition 88, 1733S-1737S. https://doi.org/10.3945/ajen.2008.25825D

[17] Botezelli, R.F., Moura, R.F., Rossi, C.A., et al. (2010) Fructose Consumption and Physical Exercise, Impact on the Metabolic Syndrome. [Consumo de frutose e exercício físico, impacto na síndrome metabolic.] Motriz, Rio Claro, 16, 231-239. 